\title{
Next-Generation Sequencing in Gynaecological Tumours: The Prognostic and Predictive Value of the Most Common Mutations Found in Ovarian, Endometrial, and Cervical Tumours: Literature Review and the University Medical Centre Utrecht Next-Generation Sequencing Data
}

\author{
Eline van Winkel ${ }^{\mathrm{a}}$ Wendy W.J. de Leng ${ }^{\mathrm{b}}$ Petronella O. Witteveen ${ }^{\mathrm{a}}$ \\ Trudy Jonges $^{\mathrm{b}}$ Stefan M. Willems ${ }^{\mathrm{b}}$ Marlies H.G. Langenberg ${ }^{\mathrm{a}}$ \\ a Department of Oncology, UMC Utrecht Cancer Center, University Medical Center Utrecht, Utrecht, The \\ Netherlands; ${ }^{b}$ Department of Pathology, University Medical Center Utrecht, Utrecht, The Netherlands
}

\section{Keywords}

Next-generation sequencing · Mutation · Gynaecological tumours · Ovarian tumours · Endometrial tumours · Cervical tumours - Biomarker - Prognostic value - Predictive value

\footnotetext{
Abstract

Objective: To investigate whether next-generation sequencing (NGS) in ovarian and endometrial tumours can discover mutations with a relevant prognostic or predictive value. Methods: After a literature search, selected studies were critically appraised using the Quality in Prognostic Studies tool. Data on mutation incidence and correlations with prognostic and predictive items were extracted from relevant studies and compared to our own cohort consisting of 28 patients analysed using NGS. Results: Eight out of 739 articles were found eligible, including different tumour types. Prevalence of mutations in the KRAS gene ranged between 5.34 and $58.8 \%$ in ovarian cancer. Two studies showed a significant
}

๑) 2017 S. Karger AG, Basel

E-Mail karger@karger.com www.karger.com/pat correlation between KRAS mutations and an improved disease free- and overall survival. Clinical data were available for 17 of our patients, mostly cases of endometrial carcinomas. KRAS, PIK3CA, CTNNB1, and TP53 were the most frequently mutated genes in endometrial carcinomas, and PTEN and CTNNB 1 correlated with a higher FIGO stage. Conclusion: In the ovary KRAS mutation is associated with type I ovarian tumours (low-grade serous, mucinous, endometrioid, and clear-cell) and may seem to have a more favourable prognosis. The prognostic value of TP53 is still controversial. In endometrial tumours, PTEN shows a positive correlation with better prognosis. PIK3CA may have a correlation with poorer prognosis. CTNNB1 mutations in endometrial carcinomas could predict a worse prognosis.

(c) 2017 S. Karger AG, Basel

E.W., W.W.J.L., S.M.W., and M.H.G.L. contributed equally to this work.
M. Langenberg

Department of Oncology, UMC Utrecht Cancer Center

University Medical Center Utrecht, Heidelberglaan 100

NL-3584CX Utrecht (The Netherlands)

E-Mail M.Langenberg@ umcutrecht.nl 


\section{Introduction}

In the field of cancer research, the use of next-generation sequencing (NGS) has increased in the last decade. As cancer is driven by heritable or somatic mutations, DNA sequencing has an important role in detecting new ways of diagnosing, predicting, and treatment of diverse cancers [1]. Sequencing of mutations in diverse cancers shows the potential of discovering new diagnostic, prognostic, therapeutic, or predictive mutation statuses. For melanoma, breast, and colon carcinomas, NGS is widely used to investigate new therapeutic strategies and to better predict prognosis [2-4]. In gynaecological tumours, the role of specific mutations is not yet thoroughly investigated, despite the widespread incidence, as gynaecological tumours account for an estimated $16 \%$ of all newly diagnosed carcinomas in women worldwide. For ovarian and endometrial cancer, 239,000 and 320,000 cases were newly diagnosed, and 152,000 and 76,000 deaths, respectively [5].

Because of the high incidence and mortality rates, but the establishment of new targeted/immunotherapy options, it is important to keep investigating new markers for prognosis, therapeutic options, and predictors of disease-free survival (DFS) or overall survival (OS). In the last few years, studies concerning somatic mutations and their role in personalized, targeted medicine in gynaecological cancers have been studied [6,7], but not as thoroughly as studies relating to breast and colon carcinomas.

The aim of this study is to explore whether NGS in ovarian and endometrial tumours can discover mutations with a relevant prognostic or predictive value in the clinical setting, by giving an overview of the evidence currently available regarding the prognostic and predictive value of mutations in ovarian and endometrial tumours and by adding new clinical data from a data set of the University Medical Centre Utrecht (UMCU).

\section{Materials and Methods}

\section{Search Strategy}

A literature search was conducted on November 6, 2015, in PubMed, Embase, and Cochrane Library. Search terms "gynaecological cancer," "next generation sequencing," and "biomarker" were combined in the search engines with the associated synonyms and Mesh and Emtree terms: "sequence analysis," "ovarian neoplasms," "uterine neoplasms," "endometrial neoplasms," "fallopian tube neoplasms," and "biological markers" (online suppl. Table 1; see www.karger.com/doi/10.1159/000479797 for all online suppl. material). Duplicates were removed, and remaining articles were screened on title and abstract, and selected or rejected accord- ing to predefined exclusion criteria (Fig. 1). Subsequently, the full text of the articles was screened. Further selection was based on an analysis of the relevance of the full text for the aim of this article, according to the criteria explained below.

\section{Eligibility Criteria and Information Sources}

Studies were found eligible for inclusion if they were written in English, were original research studies with new results on mutation analysis in any type of gynaecological cancers, and had a prognostic and/or predictive study design. Mutation analysis was required to be performed using next-generation and Sanger sequencing analysis.

Studies were excluded if they had no prognostic, predictive value linked to the mutation status, or when techniques other than sequencing were used to determine the mutation status. Studies on epigenetics, gene amplification, translocations, mRNA, or protein expression were also excluded (Fig. 1).

\section{Quality Assessment of Individual Studies}

Using criteria of the Quality in Prognosis Studies tool (QUIPS) [8], the methodological quality of the remaining articles was assessed [9].

According to the QUIPS assessment tool the following 6 items were scored as low, moderate, or high risk of bias: study participation, study attrition, prognostic factor measurement, outcome measurement, study confounding, and statistical analysis and reporting. Studies that scored a low risk of bias at 3 or more items were found to be of high quality, studies with 3 or more high risk of bias scores were found to be of low quality. Studies that scored in between these criteria were found to be of moderate quality.

\section{Data Collection and Data Items of Included Studies}

For the included articles the following items were extracted from each study, if available: study design, sample size, patient material - fresh frozen or formalin-fixed, paraffin-embedded -, tumour type, genes sequenced, sequence technique, mutation prevalence, statistical analysis, correlation with tumour grade, FIGO stage, histology, and survival outcomes.

\section{Collection and Data Extraction Samples from the UMCU \\ Database}

All endometrial and ovarian cancer samples from our sequence database (UMCU, Department of Pathology) were extracted. Samples dated between October 30, 2013, and October 28, 2015. The following items of clinical data were extracted from the patient files: date of birth, tumour type, histology and grade, FIGO stage, treatment, response to treatment, progression-free survival (PFS) and OS.

\section{DNA Extraction and Ion Torrent Personal Genome Machine} Sequencing

The tumour percentage was determined by an experienced pathologist (S.M.W.). DNA was extracted from an area containing $>10 \%$ tumour cells using ProtK and subsequent purification (Cobas, Roche). For library preparation, 20 ng DNA was amplified by multiplex PCR, targeting regions of interest using Ion AmpliSeq Cancer Hotspot Panels v2 (Thermo Fisher) or OAv2 [10] (see online suppl. Table 2 for included genes). An NGS library was prepared using the Ion Ampliseq Library Kit (Thermo Fisher) and enriched using the Ion PGM ${ }^{\mathrm{TM}}$ Template OT2 200 Template Kit 
Fig. 1. Results of the literature search: flow chart of November 6, 2015.

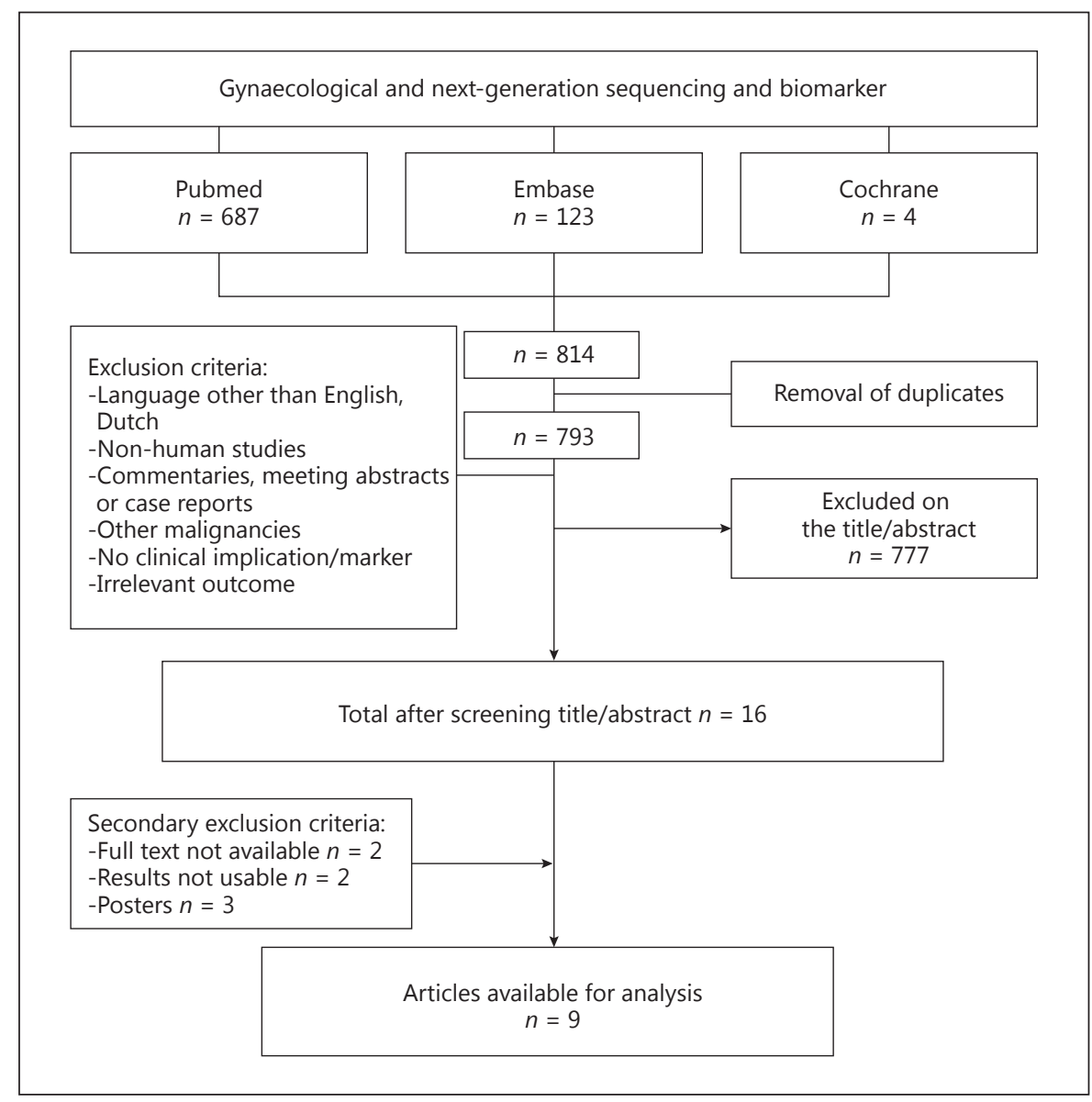

(Thermo Fisher). Sequencing was performed using the Ion PGM $^{\mathrm{TM}}$ Sequencing 200 kit v2 and the Ion Torrent PGM machine (Thermo Fisher). All procedures were performed as described by the manufacturer. The Torrent Variant Caller combined with an in-house data analysis pipeline provided filtering and annotation of sequencing data as described previously [11]. No germline, Lynch syndrome, or BRCA1/2 analysis was performed.

\section{Synthesis of Results}

Because of the heterogeneity of the cohorts, the analysis performed, and the results, a pooled analysis of the results was not possible. All individual results are shown. PFS was defined as from time of diagnosis until progression; OS was defined as from time of diagnosis until last contact.

\section{Results}

\section{Search and Selection}

The initial search yielded 793 unique articles. Sixteen articles were selected for full-text screening of which 2 were not available in full text, and 3 were posters. Another article had a discordant domain and 1 did not contain sequence data. Eight of the articles were considered eligible for final analysis after screening [12-19]. Six of them reported on ovarian cancer $[12-15,18,19]$, and 2 papers investigated endometrial cancer [16, 17] (Fig. 1; Table 1).

\section{Quality Assessment}

The quality of all 8 studies was assessed using the QUIPS tool (Table 1). Seven were relevant to our study and the eighth [17] did not describe a prognostic or predictive outcome, but the determinant and domain were the same as in our research question. Therefore this paper was also included for further analysis.

The quality of the included papers ranged between good [14, 16, 17] and moderate [12, 13, 15, 18, 19]. Overall the articles were according to our research question and of fairly good quality. In particular the item "study confounding" scored insufficient, as the papers did not describe confounding factors, or a statistical analysis, whereby confounding factors were taken into account, was lacking (Table 1). Study attrition was scored moderate or high risk of bias, as in the concerned papers loss to 
Table 1. Quality assessment of studies using the Quality in Prognosis Studies (QUIPS) assessment tool

\begin{tabular}{|c|c|c|c|c|c|c|}
\hline Tumor type and study & $\begin{array}{l}\text { Study par- } \\
\text { ticipation }\end{array}$ & $\begin{array}{l}\text { Study } \\
\text { attrition }\end{array}$ & $\begin{array}{l}\text { Prognostic } \\
\text { factor } \\
\text { measurement }\end{array}$ & $\begin{array}{l}\text { Outcome } \\
\text { measurement }\end{array}$ & $\begin{array}{l}\text { Study con- } \\
\text { founding }\end{array}$ & $\begin{array}{l}\text { Statistical } \\
\text { analysis and } \\
\text { reporting }\end{array}$ \\
\hline \multicolumn{7}{|l|}{ Ovarian cancer } \\
\hline Despierre et al. [14], 2014 & O & O & O & ○ & (1) & O \\
\hline Jia et al. [18], 2014 & D & D & 0 & 0 & (1) & D \\
\hline Anglesio et al. [12], 2013 & (1) & (1) & 0 & O & O & $\bigcirc$ \\
\hline Dobrzycka et al. [15], 2011 & $\bigcirc$ & 0 & O & ○ & (1) & (1) \\
\hline Bauerschlag et al. [13], 2010 & (1) & D & D & ○ & O & $\bigcirc$ \\
\hline Wong et al. [19], 2010 & (1) & D & 0 & 0 & D & 0 \\
\hline \multicolumn{7}{|l|}{ Endometrial cancer } \\
\hline Garcia-Dios et al. [16], 2013 & 0 & D & 0 & O & 0 & $\bigcirc$ \\
\hline Gatius et al. [17], 2011 & $\bigcirc$ & D & 0 & O & $\mathrm{O}$ & $\bigcirc$ \\
\hline
\end{tabular}

The articles included were assessed on quality using the QUIPS tool. A comprehensive description of each QUIPS item is provided by Hayden et al. [8]. Risk of bias: $\bigcirc$, low; $\mathbf{O}$, moderate; $\boldsymbol{O}$, high.

follow-up was often not mentioned. All papers used welldefined prognostic factor measurements as well as outcome measurements. No studies were excluded based on the quality assessment.

\section{Study Characteristics}

The included studies comprised a total of $1,997 \mathrm{pa}-$ tients. Seven studies made prognostic correlations, and 3 had predictive conclusions as well (Table 2). As mentioned before, Gatius et al. [17] did not make any prognostic or predictive conclusions. Of the 6 papers concerning ovarian cancer, 1 made a distinction between lowgrade serous carcinomas, high-grade serous carcinomas and serous borderline tumours [19]. The other 5 discriminated between type I tumours (low-grade serous carcinomas, mucinous, endometrioid, and clear-cell carcinomas) and type II tumours (high-grade serous carcinomas, undifferentiated cancers and carcinosarcomas). KRAS was the most frequently investigated gene, followed by PIK3CA. The material used for sequencing, formalinfixed, paraffin-embedded $[12,17,18]$, fresh frozen $[15$, 19], or both $[13,14,16]$ differed among the studies (Table 2).

\section{Literature Study Results}

Ovarian Cancer

The prevalence of mutations in the KRAS gene ranged between 2.9 and $49 \%$ in ovarian cancer $[12,14]$. TP53 mutations detected by sequencing methods were found to be $57.5 \%$ in the study of Bauerschlag et al. [13] and 73\% in high-grade serous carcinoma in the study of Wong et al. [19]. The mutation prevalences of the other studies are shown in Table 3.

KRAS mutations were associated with (1) histological type, (2) tumour grade, (3) response to therapy, and (4) clinical outcome. Most studies showed a correlation between KRAS mutation and mucinous tumour type. Mutation frequencies ranged from $23.5 \%$ [18] to 50\% [14]. KRAS mutations were most common in type I tumours [18]: $27 \%$ of grade I tumours [14] and 19\% of the lowgrade serous carcinomas [19], although this could not be confirmed by another study [15]. Two studies showed a significant correlation between KRAS mutations and an improved DFS, PFS, and OS $[12,18]$ although Dobrzycka et al. [15] and Despierre et al. [14] did not find a significant correlation between all these parameters.

In the study of Despierre et al. [14] neither KRAS nor PIK3CA mutations had a significant effect on the response to platinum-based chemotherapy. Despierre et al. found that PIK3CA is most common in tumours with FIGO stage Ia-IIc, and that the prevalence is the highest in clear-cell carcinomas (46.2\%). Bauerschlag et al. [13] did not report any data on KRAS mutations but looked into TP53 mutations in ovarian cancer instead. They showed that the TP53 mutation rate increases when cell differentiation decreases. No differences were found between early- and late-stage ovarian cancer and histological subtypes, and no correlation between TP53 status, platinum response, 2-year survival and OS was seen. 
Table 2. Study characteristics of selected articles

\begin{tabular}{|c|c|c|c|c|c|c|c|}
\hline $\begin{array}{l}\text { Despierre et al. } \\
{[14], 2014}\end{array}$ & $\begin{array}{l}\text { Prognostic, } \\
\text { predictive }\end{array}$ & 262 & $\begin{array}{l}\text { Serous, mucinous, } \\
\text { clear-cell, } \\
\text { endometrioid, other } \\
\text { ovarian tumours }\end{array}$ & $\begin{array}{l}\text { KRAS, PIK3CA, } \\
\text { BRAF, NRAS, } \\
\text { PTEN, FBXW7, } \\
\text { AKT2, AKT3, } \\
\text { FOXL2 }\end{array}$ & $\begin{array}{l}\text { FF, } \\
\text { FFPE }\end{array}$ & $\begin{array}{l}\text { Sequenom } \\
\text { MassARRAY }\end{array}$ & $\begin{array}{l}\text { Friedman } \chi^{2}, \text { Cochran- } \\
\text { Mantel-Haenszel test, } \\
\text { Kaplan-Meier curves, } \\
\text { partial Spearman } \\
\text { correlation coefficient }\end{array}$ \\
\hline $\begin{array}{l}\text { Anglesio et al. } \\
{[12], 2013}\end{array}$ & $\begin{array}{l}\text { Prognostic, } \\
\text { predictive }\end{array}$ & 104 & $\begin{array}{l}\text { Mucinous, mucinous } \\
\text { borderline ovarian } \\
\text { tumours }\end{array}$ & $\begin{array}{l}\text { KRAS (NRAS, } \\
\text { BRAF, ERBB2) }\end{array}$ & FFPE & $\begin{array}{l}\text { Sanger sequencing, } \\
\text { ABI Prism 3130xl } \\
\text { genetic analyser }\end{array}$ & $\begin{array}{l}\text { Kaplan-Meier curves, } \\
\text { log-rank statistic, Cox } \\
\text { proportional hazards } \\
\text { model }\end{array}$ \\
\hline $\begin{array}{l}\text { Bauerschlag } \\
\text { et al. } \\
{[13], 2010}\end{array}$ & $\begin{array}{l}\text { Prognostic, } \\
\text { predictive }\end{array}$ & 104 & $\begin{array}{l}\text { Serous, mucinous, } \\
\text { clear-cell, endometrioid } \\
\text { ovarian tumours }\end{array}$ & TP53 & $\begin{array}{l}\text { FF, } \\
\text { FFPE }\end{array}$ & $\begin{array}{l}\text { Dideoxy sequencing } \\
\text { using a T7 sequencing } \\
\text { kit }\end{array}$ & $\chi^{2}$ test, Fisher exact test \\
\hline $\begin{array}{l}\text { Wong et al. } \\
\text { [19], } 2010\end{array}$ & Prognostic & 91 & $\begin{array}{l}\text { Low-grade, high-grade, } \\
\text { borderline serous } \\
\text { ovarian tumours }\end{array}$ & $\begin{array}{l}\text { BRAF, KRAS, } \\
\text { TP53 }\end{array}$ & $\mathrm{FF}$ & $\begin{array}{l}\text { SNP array, Affymetris } \\
\text { Fluidic Station } 450 \text {, } \\
\text { Gene chip scanner } \\
3000\end{array}$ & $\begin{array}{l}\text { Kaplan-Meier curves, } \\
\text { log-rank statistic }\end{array}$ \\
\hline $\begin{array}{l}\text { Garcia-Dios } \\
\text { et al. } \\
\text { [16], } 2013\end{array}$ & Prognostic & 1,063 & $\begin{array}{l}\text { Endometrial carcinoma } \\
\text { type I, II }\end{array}$ & $\begin{array}{l}\text { PTEN, PIK3CA, } \\
\text { KRAS, TP53, } \\
\text { FBXW7, NRAS }\end{array}$ & $\begin{array}{l}\text { FF, } \\
\text { FFPE }\end{array}$ & $\begin{array}{l}\text { iPLEX technology, } \\
\text { MassARRAY } \\
\text { compact analyser }\end{array}$ & $\begin{array}{l}\text { Binary logistic regression, } \\
\text { Cox proportional hazards } \\
\text { model }\end{array}$ \\
\hline
\end{tabular}

FF, fresh frozen tumour tissues; FFPE, formalin-fixed, paraffin-embedded tumour tissues.

Wong et al. [19] reported a prevalence of TP53 mutations in $73 \%$ of the high-grade serous carcinoma and $0 \%$ in low-grade or borderline tumours.

\section{Endometrial Cancer}

Garcia-Dios et al. [16] and Gatius et al. [17] investigated different genes in endometrial cancer. Gatius et al. reported an FGFR2 mutation prevalence of $6.45 \%$ and found that these mutations only occur in endometrioid carcinoma. They described that FGFR2 may be a target for therapeutic intervention. For other genes such as PTEN, KRAS, and PIK3CA, Gatius et al. [17] reported a prevalence of 48,23 , and $29 \%$, respectively, whereas Garcia-Dios et al. [16] reported a prevalence of 15.4, 15.1, and $16.2 \%$, respectively. Furthermore, a correlation between
TP53 mutation and type II tumours (serous, clear-cell carcinomas) and PTEN mutation with type I tumours (endometrioid carcinomas) was found [16]. PIK3CA correlated with grade 2-3 tumours. A correlation between mutation status and recurrence was not reported. Only PIK3CA hot spot mutation H1047R correlated significantly with a poorer prognosis.

\section{Characteristics of UMCU Data}

Our data set consisted of 20 tumour samples the mutation status of which was analysed by NGS. Of these, 17 consisted of endometrial cancers and 3 of cervical cancer (Fig. 2). From 14 patients we had clinical data consisting of tumour histology, grading, FIGO stage, treatment, and follow-up data (Table 4). Of the patients with clinical 
Table 3. Study results of selected articles

\begin{tabular}{|c|c|c|c|c|c|c|}
\hline Study & $\begin{array}{l}\text { Tu- } \\
\text { mour } \\
\text { type }\end{array}$ & Genes & $\begin{array}{l}\text { Patients } \\
\text { with mu- } \\
\text { tation, } n\end{array}$ & Mutation prevalence & $\begin{array}{l}\text { Prognostic outcomes (correlation with } \\
\text { FIGO stage/grade/histology/PFS/OS) }\end{array}$ & Predictive outcomes \\
\hline $\begin{array}{l}\text { Jia et al. } \\
{[18], 2014}\end{array}$ & OC & KRAS & 16 & $14.7 \%$ & $\begin{array}{l}\text { KRAS mutation associated with lower grade } \\
23.5 \% \text { of the mucinous tumours harboured } \\
\text { KRAS mutations } \\
\text { Tumours harbouring KRAS mutations have } \\
\text { probably a more favourable prognosis }\end{array}$ & - \\
\hline $\begin{array}{l}\text { Dobrzycka } \\
\text { et al. } \\
{[15], 2011}\end{array}$ & OC & KRAS & 27 & $21.4 \%$ & $\begin{array}{l}61.1 \% \text { of mucinous, } 23.1 \% \text { of endometrioid, } \\
12.5 \% \text { of serous OCs harboured KRAS } \\
\text { mutations } \\
\text { KRAS mutations were associated with grade } \\
1 \text { tumours } \\
\text { KRAS mutations may predict an improved } \\
\text { OS in mucinous carcinomas }\end{array}$ & - \\
\hline $\begin{array}{l}\text { Bauerschlag } \\
\text { et al. } \\
\text { [13], } 2010\end{array}$ & OC & TP53 & $27(42)^{2}$ & $26 \%(57.5 \%)$ & $\begin{array}{l}\text { TP53 mutation correlates with de- } \\
\text { differentiation } \\
\text { No correlation between TP53 mutation, } \\
\text { FIGO stage or histological subtypes }\end{array}$ & $\begin{array}{l}\text { TP53 mutation showed } \\
\text { no correlation with the } \\
\text { response to platinum- } \\
\text { based chemotherapy, } \\
\text { or } 2 \text {-year survival }\end{array}$ \\
\hline $\begin{array}{l}\text { Gatius et al. } \\
{[17], 2011}\end{array}$ & $\mathrm{EC}$ & $\begin{array}{l}\text { FGFR2 }(P T E N, \\
\text { KRAS, CTNNB1, } \\
\text { PIK3CA })^{3}\end{array}$ & 2 & $\begin{array}{l}\text { FGFR2 6.45\% (PTEN } \\
48 \%, \text { KRAS 23\%, } \\
\text { CTNNB1 17\%, PIK3CA } \\
29 \%)\end{array}$ & - & - \\
\hline
\end{tabular}

OS, overall survival; DFS, disease-free survival; OC, ovarian carcinoma; LGSC, low-grade serous carcinoma; SBOT, serous borderline tumour; HGSC, high-grade serous carcinoma; EC, endometrial cancer; OS, overall survival; PFS, progression-free survival. ${ }^{1}$ In mucinous tumours, when assessed within mucinous carcinomas, no association was demonstrated. ${ }^{2}$ Authors defined 42 cases as mutated by different techniques, 27 of them were confirmed by sequencing. ${ }^{3}$ Genes evaluated previously in the same cases. 
Fig. 2. Mutation prevalence in UMCU patients.

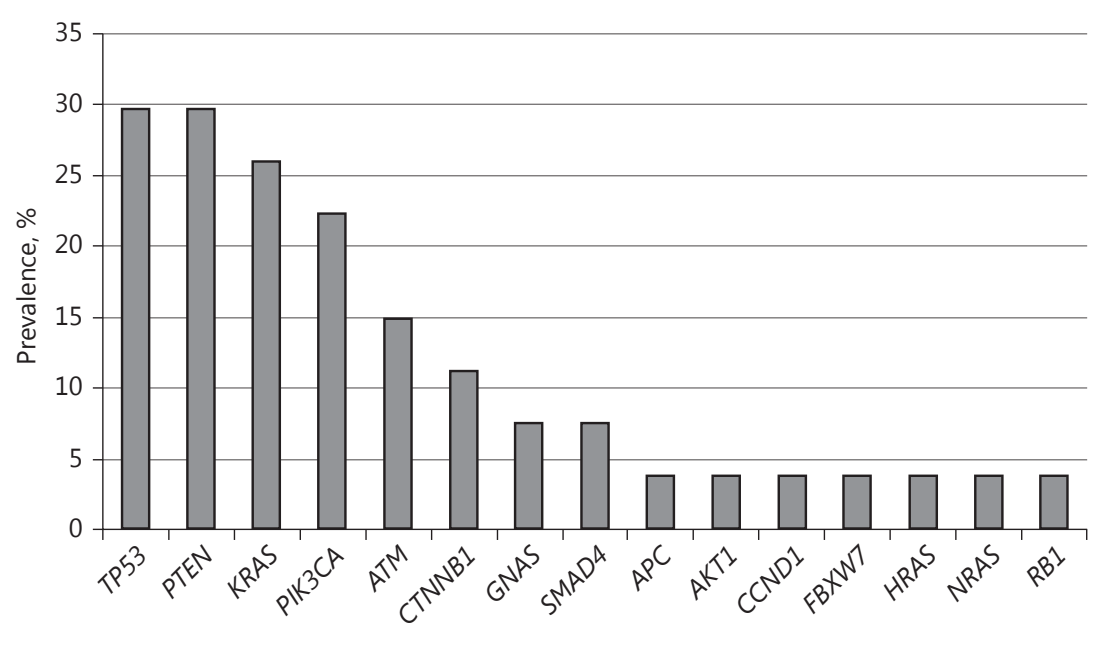

Fig. 3. Mutation prevalence of different genes per tumour type in UMCU patients.

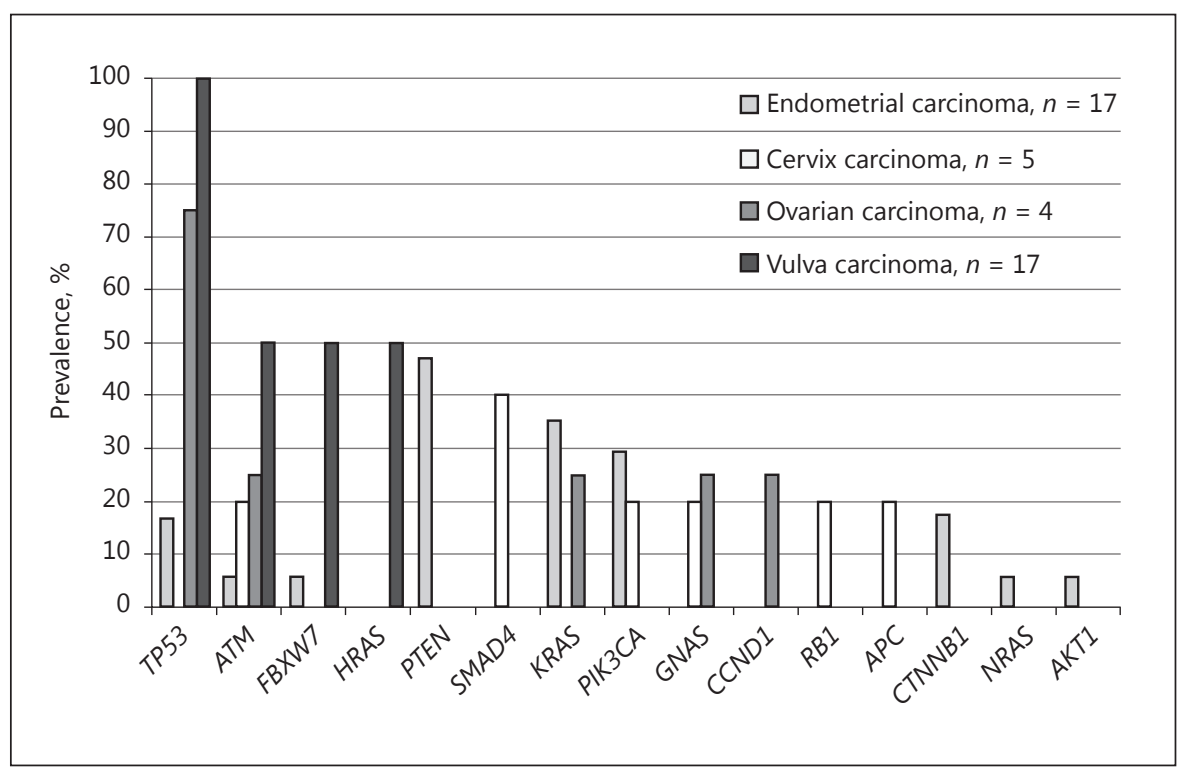

data, 2 were diagnosed with ovarian cancer, 12 with endometrial cancer.

\section{Results of UMCU Data}

TP53 was mutated in $100 \%$ of the ovarian tumours (Fig. 2, 3). CCND1 was mutated in $33 \%$ of the cases. Endometrial tumours comprised the largest group within the cohort and showed a PTEN mutation rate of $47 \%$; KRAS, PIK3CA, CTNNB1, and TP53 mutations showed a prevalence of $35,29,18$, and $18 \%$, respectively.
Clinical data were available in 12 cases (Table 4). In endometrial tumours, TP53 mutations only occurred in FIGO stage Ia-b, 2 occurred in non-endometrioid carcinomas, the other sample with a TP53 mutation was a mixed tumour. PIK3CA mutations were only identified in low FIGO stages Ia-c. Two of the 3 KRAS mutations occurred in FIGO stage Ia-b. Higher FIGO stage tumours correlated with PTEN and CTNNB1 mutations. All CTNNB1 mutations occurred in FIGO stage IIIa-b and 3 out of 4 PTEN mutations in FIGO stage IIIa. PTEN muta- 
Table 4. Results of the UMCU cohort

\begin{tabular}{|c|c|c|c|c|c|c|c|c|c|c|}
\hline $\begin{array}{l}\mathrm{Pa}- \\
\text { tient }\end{array}$ & $\begin{array}{l}\text { Tumour } \\
\text { type }\end{array}$ & Mutation & Histology & Grade & $\begin{array}{l}\text { FIGO } \\
\text { stage }\end{array}$ & Primary treatment & $\begin{array}{l}\text { Response/ } \\
\text { PFS }\end{array}$ & $\begin{array}{l}\text { Next lines } \\
\text { of therapy }\end{array}$ & $\begin{array}{l}\text { Response/ } \\
\text { PFS }\end{array}$ & OS \\
\hline 1 & Ovarian & $T P 53$, c.637C $>\mathrm{T}$ & Carcinosarcoma & - & IIIC & $\begin{array}{l}\text { Optimal debulking, } \\
\text { carboplatin/paclitaxel }\end{array}$ & $\begin{array}{l}\text { CR, } \\
11 \text { months, } \\
\text { ongoing }\end{array}$ & - & - & Alive \\
\hline $2^{1}$ & Ovarian & $\begin{array}{l}C C N D 1, \text { c. } 790 \mathrm{C}>\mathrm{T} \\
T P 53, \text { c. } 1004 \mathrm{G}>\mathrm{A}\end{array}$ & $\begin{array}{l}\text { Clear-cell } \\
\text { adenocarcinoma }\end{array}$ & - & IIb & $\begin{array}{l}\text { Debulking, RTx, carboplatin/ } \\
\text { paclitaxel }\end{array}$ & $\begin{array}{l}\mathrm{U}, \\
24 \text { months }\end{array}$ & $\begin{array}{l}2 \text { times local } \\
\text { surgery }\end{array}$ & $\begin{array}{l}\mathrm{U}, \\
4 \text { months, } \\
6 \text { months }\end{array}$ & Alive \\
\hline 3 & Ovarian & TP53, c.242delC & Serous carcinoma & - & - & - & - & - & - & - \\
\hline 4 & Uterus & $\begin{array}{l}C T N N B 1, \text { c. } 110 \mathrm{C}>\mathrm{T} \\
P T E N, \text { c } 540 \mathrm{C}>\mathrm{A}\end{array}$ & $\begin{array}{l}\text { Endometrioid } \\
\text { adenocarcinoma }\end{array}$ & 2 & IIIa & $\begin{array}{l}\text { Hysterectomy with adnexa, } \\
\text { RTx }\end{array}$ & $\begin{array}{l}\text { NED, } \\
2 \text { months, } \\
\text { ongoing }\end{array}$ & - & - & Alive \\
\hline 5 & Uterus & $\begin{array}{l}\text { KRAS, с. } 34 \mathrm{G}>\mathrm{T} \\
P T E N, \text { с.389G }>\mathrm{A}\end{array}$ & $\begin{array}{l}\text { Endometrioid } \\
\text { adenocarcinoma }\end{array}$ & 2 & IIIa & $\begin{array}{l}\text { Hysterectomy with adnexa, } \\
\text { RTx }\end{array}$ & $\begin{array}{l}\mathrm{U}, \\
2 \text { months, } \\
\text { ongoing }\end{array}$ & - & - & Alive \\
\hline $6^{1}$ & Uterus & $C C N D 1$, c.790C $>\mathrm{T}$ & $\begin{array}{l}\text { Clear-cell } \\
\text { adenocarcinoma }\end{array}$ & - & II & $\begin{array}{l}\text { Debulking, RTx, carboplatin/ } \\
\text { paclitaxel }\end{array}$ & $\begin{array}{l}\mathrm{U}, \\
24 \text { months }\end{array}$ & $\begin{array}{l}2 \text { times local } \\
\text { surgery }\end{array}$ & $\begin{array}{l}\mathrm{U}, \\
4 \text { months, } \\
6 \text { months }\end{array}$ & Alive \\
\hline 7 & Uterus & $\begin{array}{l}\text { KRAS, c.35G }>\text { A } \\
P T E N, \text { c.951_954del4 } \\
\text { TP53, c.637C }>\mathrm{T}\end{array}$ & $\begin{array}{l}\text { Endometrioid/ } \\
\text { carcinosarcoma }\end{array}$ & $2 / 3$ & $\mathrm{Ib}$ & $\begin{array}{l}\text { Hysterectomy with adnexa } \\
\text { and appendix }\end{array}$ & $\mathrm{U}$ & $\mathrm{U}$ & $\mathrm{U}$ & Alive \\
\hline 8 & Uterus & $\begin{array}{l}\text { PIK3CA, c.1633G }>A \\
\text { TP53, c.560-1G>C }\end{array}$ & $\begin{array}{l}\text { Clear-cell } \\
\text { carcinoma }\end{array}$ & - & Ia & $\begin{array}{l}\text { Hysterectomy with adnexa, } \\
\text { para-aortal LND, } \\
\text { omentectomy }\end{array}$ & $\begin{array}{l}\text { NED, } \\
20 \text { months, } \\
\text { ongoing }\end{array}$ & - & - & Alive \\
\hline 9 & Uterus & $\begin{array}{l}A K T 1, \mathrm{c} .49 \mathrm{G}>\mathrm{A} \\
C T N N B 1, \mathrm{c} .110 \mathrm{C}>\mathrm{T} \\
F B W X 7, \mathrm{c} .1393 \mathrm{C}>\mathrm{T}\end{array}$ & Endometrioid & 1 & IIIb & $\begin{array}{l}\text { Hysterectomy with adnexa, } \\
\text { RTx }\end{array}$ & $\begin{array}{l}\text { NED, } \\
16 \text { months, } \\
\text { ongoing }\end{array}$ & - & - & Alive \\
\hline 10 & Uterus & KRAS, c.35G>A & Endometrioid & 1 & Ia & Hysterectomy with adnexa & $\begin{array}{l}\mathrm{U}, \\
19 \text { months }\end{array}$ & $\begin{array}{l}\text { RTx; } \\
\text { medroxy- } \\
\text { progesterone }\end{array}$ & $\begin{array}{l}\mathrm{RD}, 8 \text { months; } \\
\mathrm{SD}, 5 \text { months, } \\
\text { ongoing }\end{array}$ & Alive \\
\hline 11 & Uterus & $\begin{array}{l}\text { NRAS, c. } 182 \mathrm{~A}>\mathrm{G} \\
P I K 3 C A, \mathrm{c} .1637 \mathrm{~A}>\mathrm{G}\end{array}$ & Endometrioid & 2 & Ic & $\begin{array}{l}\text { Hysterectomy with adnexa, } \\
\text { RTx }\end{array}$ & $\begin{array}{l}\text { NED, } \\
96 \text { months }\end{array}$ & $\begin{array}{l}\text { Colon } \\
\text { carcinoma }\end{array}$ & & Alive \\
\hline 12 & Uterus & $\begin{array}{l}\text { PIK3CA, c. } 3140 \mathrm{~A}>\mathrm{G} \\
\text { TP53, c. } 577 \mathrm{C}>\mathrm{T}\end{array}$ & Carcinosarcoma & 3 & $\mathrm{Ib}$ & $\begin{array}{l}\text { Hysterectomy, cisplatin/ } \\
\text { epirubicin, RTx }\end{array}$ & $\begin{array}{l}\text { NED, } \\
36 \text { months, } \\
\text { ongoing }\end{array}$ & - & - & Alive \\
\hline 13 & Uterus & $\begin{array}{l}\text { CTNNB1, c. } 110 \mathrm{C}>\mathrm{T} \\
\text { PTEN, c. } 540 \mathrm{C}>\mathrm{A}\end{array}$ & Endometrioid & 2 & IIIa & $\begin{array}{l}\text { Hysterectomy with adnexa, } \\
\text { RTx }\end{array}$ & $\begin{array}{l}\text { NED, } \\
12 \text { months } \\
\text { ongoing }\end{array}$ & - & - & Alive \\
\hline 14 & Uterus & $\begin{array}{l}\text { KRAS, c. } 35 \mathrm{G}>\mathrm{A} P T E N, \\
\text { c. } 29 \mathrm{G}>\mathrm{A}\end{array}$ & $\begin{array}{l}\text { Endometrioid } \\
\text { adenocarcinoma }\end{array}$ & - & - & - & - & - & - & - \\
\hline 15 & Uterus & - & $\begin{array}{l}\text { Endometrioid } \\
\text { adenocarcinoma }\end{array}$ & - & - & - & - & - & - & - \\
\hline 16 & Uterus & $\begin{array}{l}\text { KRAS, c. } 34 \mathrm{G}>\mathrm{T} P T E N, \\
\text { c. } 389 \mathrm{G}>\mathrm{A}\end{array}$ & Adenocarcinoma & - & - & - & - & - & - & - \\
\hline 17 & Uterus & PTEN, c. $388 \mathrm{C}>\mathrm{G}$ & Adenocarcinoma & - & - & - & - & - & - & - \\
\hline 18 & Uterus & $P I K 3 C A, c .1637 \mathrm{~A}>\mathrm{G}$ & Adenocarcinoma & - & - & - & - & - & - & - \\
\hline 19 & Uterus & $\begin{array}{l}\text { KRAS, c.35G }>\text { A PIK3CA, } \\
\text { c. } 325 \_327 \mathrm{del} 3 \text { and } \\
\text { c. } 3139 \mathrm{C}>\mathrm{T} \\
P T E N, \text { c.520_528del9 } \\
\text { and c.697C }>\mathrm{T}\end{array}$ & Adenocarcinoma & - & - & - & - & - & - & - \\
\hline
\end{tabular}

UMCU, University Medical Centre Utrecht; PFS, progression-free survival; OS, overall survival; U, unknown; CR, complete remission; NED, no evidence of disease; RD, regression of disease; SD, stable disease; RTx, radiotherapy; LND, lymph node dissection. ${ }^{1}$ Same patient, 2 primary tumours.

tions only occurred in grade 2 tumours and endometrioid carcinomas. No correlation was seen with treatment, PFS and OS. For ovarian carcinomas, no correlation between clinical data and mutation status was seen, most probably due to small sample sizes.

\section{Discussion}

In this paper we investigated whether NGS can discover mutations in gynaecological tumours with relevant prognostic or predictive value. A literature search was conducted in order to give an overview of the evidence currently available regarding the prognostic and predic- 
tive value of mutations in gynaecological tumours. In an attempt to improve our knowledge of the value of the mutational status of gynaecological cancers, new clinical data on this subject from a data set of the UMCU was added.

\section{Ovarian Carcinomas, TP53 and KRAS Mutations}

In a large study of Spaans et al. [20], PIK3CA, PTEN, and KRAS were found the most frequently occurring mutations in gynaecological carcinomas in 22,18 and $12 \%$, respectively. This is comparable with the results of our study where 40,30 , and $25 \%$ of tumours harboured a PTEN, KRAS, or PIK3CA mutation, respectively. TP53 mutations occurred in $30 \%$ of our samples (Fig. 2) but this gene was not included in the study of Spaans et al.

Different grades of ovarian cancer have been associated with different genetic alterations [21]. In type I tumours (low-grade serous, mucinous, endometrioid, clear-cell) KRAS and BRAF are often mutated [12, 14, 22] whereas TP53 mutations are rare; type II tumours (highgrade serous, undifferentiated cancers, carcinosarcomas) on the other hand harbour often TP53 mutations [22,23]. TP53 mutation frequencies have been described in highgrade tumours occurring in 58 [13], 73 [19], and 96\% [24]. We identified a TP53 mutation frequency in ovarian tumours of $100 \%$, but note that the sample size was only 3.

Since the TP53 mutation status is linked to increased genetic instability as DNA replication is no longer paused when errors occur, an adverse effect on the efficacy of platinum-based DNA intercalating substrates was suggested. Since resistance to platinum-based therapy is a problem in the treatment of ovarian cancer, TP53 mutation status could serve as a predictive marker. Unfortunately, the link between TP53 mutation status and response to platinum-based chemotherapy remains unclear. Reles et al. [21] showed a significant correlation between TP53 mutation and platinum resistance, although its significance was lost in a multivariate analysis. In contrast, Bauerschlag et al. [13] could not detect a correlation using different methods to detect TP53 mutations, thereby excluding the potential effect of mutation detection on the DNA, RNA, or protein level.

The association of KRAS mutations and prognosis is also not yet fully described in the literature $[25,26]$. Jia et al. [18] and Anglesio et al. [12] reported a significant positive correlation between the KRAS mutation status and DFS/PFS/OS. Despierre et al. [14], however, showed no correlation. Because of the very small sample size of ovarian tumours in our data set, we cannot draw any conclusions.

Next-Generation Sequencing
Endometrial Carcinomas, PIK3CA, TP53, KRAS, PTEN, and CTNNB1 Mutations

In the subgroup of endometrial carcinomas a high prevalence of PTEN, KRAS, and PIK3CA mutations was detected in this study. The most frequently mutated gene was PTEN with a mutation frequency of $47 \%$ of the endometrial carcinomas, where the literature shows a prevalence ranging from 26 to $82 \%$ [17, 20, 27-30]. PTEN mutations occur frequently in premalignant lesions and in type I endometrial cancer [27, 30, 31]. In our study, it was noted that PTEN mutations occurred only in endometrioid carcinomas and grade 2 tumours, which is similar to the existing literature $[16,27,28]$. PTEN mutations show a positive correlation with a favourable prognosis [31]. Mutations in PIK3CA, a gene within the same signalling pathway as $P T E N$, are also linked to type I endometrial carcinomas $[27,28]$. In our data set, PIK3CA mutations occurred in $29 \%$ of the cases, which is in line with previous reports ranging between 16 and $52 \%[16,17,32,33$, 34 , where the lowest frequencies can be explained by targeted sequencing (hot spot only). For PIK3CA mutations, a correlation was described with a poorer prognosis as a result of the correlation with higher tumour grade [16, 17]. In our samples this correlation was not found. Konopka et al. [33] however describe a weak correlation between PIK3CA mutations and higher tumour grade. KRAS mutations occurred in $35 \%$ of our samples, which is slightly higher than previously reported rates of 10 $31 \%[16,17,20,27,28]$. KRAS mutations were mainly detected in low FIGO stage and endometrioid carcinomas (type I tumours). Garcia-Dios et al. [16] reported no difference in KRAS mutation between the FIGO stages but a higher prevalence with type I tumours as well, corresponding with previously reported papers [27, 28]. CTNNB1 mutations in our data set were found in $18 \%$ of the cases, which correlates with previous studies $[17,20$, $27,35]$. In our data set, CTNNB1 mutations correlated with higher FIGO stage (IIIa-b) and grade 1-2 tumours; however, Saegusa et al. [36] described a correlation between CTNNB1 and lower-grade tumours. A significant correlation between CTNNB1 mutations and metastasis was described in their study, which corresponds to our finding of enrichment of CTNNB1 mutations in high FIGO staged tumours. TP53 mutations are associated with type II endometrial carcinomas [16, 27, 28]. In our data set, 2 TP53 mutations occurred in type II endometrial carcinomas and 1 in a mixed tumour type. Our data therefore also suggest a correlation between TP53 mutations and type II carcinoma, but note that this is based on only 3 tumours. No correlation between TP53 and DFS 
or OS could be demonstrated based on our findings [37, 38].

\section{Online Database}

In online supplementary Table 3 the 20 most common mutations in ovarian serous carcinomas and endometrial carcinomas according to the cBioPortal database [39] are shown. Not all of these genes were included in the gene panel used for sequencing in our cohort, as only the most common mutated genes were included. For ovarian cancer however, only 1 gene was found to overlap between our panel and the top 20 genes mutated shown in the database. For example, KRAS mutations are detected in $5.34-58.8 \%[12,14]$, whereas in the database, KRAS is only mutated in $0.4 \%$ [39]. This difference can be explained by the tumour types included in the different studies. In the papers described above, KRAS mutations were mostly associated with mucinous tumours. The data used in the database of cbioportal.org are serous carcinomas. For endometrial cancers, the mutation prevalence reported in our study and that of the database are comparable.

\section{Pathways}

The affected genes in gynaecological tumours are part of several signalling pathways, including RAS-PI3K, NOTCH and Wnt signalling [24, 40, 41]. Not only somatic mutations, but alternative mechanisms like copy number aberrations and methylation events can cause changes in these pathways eventually resulting in neoplastic transformation, for example amplifications in the RAS/PI3K pathway in ovarian cancers [24].

In high-grade serous ovarian cancer, potential treatment options exist for $45 \%$ of the cases where RAS/PI3K signalling is altered due to copy number alterations and in a smaller part by mutations [24]. The homologous recombination pathway is also affected in about $50 \%$ of the tumours providing potential treatment options with PARP inhibitors, as already applied in the clinic for BRCA mutant platinum-sensitive ovarian cancers [42]. In type I ovarian tumours the RAS pathway is frequently affected, implying a potential role for MEK inhibitors. Although some patients show a complete response [43], others do not, implying the need for identifying a clear biomarker.

In endometrial cancers the PI3K/AKT pathway is most affected where mutations in PTEN and PIK3CA lead to activation of this anti-apoptotic pathway. AKT activation can result in activation of the mTOR signalling pathway providing a rationale for treatment with MTOR inhibitors [44]. In early clinical studies benefit is shown from mTOR inhibition, but there are no results from latephase trials yet $[45,46]$. Some endometrium tumours are highly mutated caused by a mutation in POLE [40]. As highly mutated tumours are shown to respond to immunotherapy, this might provide a treatment option for this subset of tumours [47].

\section{Overall Remarks, Strengths, and Limitations}

Overall limitations of our cohort study are both the small sample size of the data set and the heterogeneity of both the samples and reviewed articles included. We want to stress that the histology as mentioned in the original paper is used in this review, which might sometimes result in unclarity regarding the exact histological subtype. Besides that, a panel-based sequencing analysis was performed, so the investigated variations were limited and no information was gained about mutation load or gene signatures, possibly predictive for immunotherapy treatment.

DNA sequencing to find predictive/prognostic correlations is a valuable technique and (for some cancers) crucial in clinical decision-making. However, it should be noted that only looking at DNA aberrations as prognostic/predictive biomarkers is rather 1-dimensional and a limitation of our research method. To overcome this weakness, a broader view is needed and other techniques involving proteomics, metabolomics, and epigenetics should be coupled to genomics to provide a multidimensional approach and also biological explanations for the found genomic variants. The strengths of our study are the thorough literature search performed, and the critical appraisal of the studies included using the QUIPS tool [8]. The distinctive search combined with our cohort and the database verification supplement each other and give a firm overview about current evidence concerning the value of different mutations in prognostic and predictive terms.

\section{Conclusion}

In this study DNA sequencing results of a literature search and our own gynaecological patient population were described. In general, our findings show similarity to the reports in the literature. In summary, PTEN, PIK3CA, KRAS, and TP53 were the most commonly mutated genes in gynaecological cancers. In ovarian tumours, KRAS is associated with type I ovarian tumours (low-grade serous, mucinous, endometrioid, and clearcell) and may seem to have a more favourable prognosis. 
TP53, on the other hand, shows a correlation with type II ovarian tumours (high-grade serous, undifferentiated cancers, carcinosarcomas). The predictive value, however, is still controversial. In endometrial tumours, PTEN is frequently mutated, especially in type I tumours (endometrioid carcinomas), and shows a positive correlation with better prognosis. PIK3CA is also correlated with type I tumours, and a correlation may exist with poorer prognosis. CTNNB1 mutations in endometrial carcinomas could predict a worse prognosis based on the correlation with higher FIGO stage and metastasis. TP53 muta- tions are correlated with type II tumours (serous, clearcell carcinomas).

For conclusions regarding clinical relevance, and for the investigation of the prognostic and predictive value of the mutations found, a prospective trial with a high number of samples is needed, in order to perform a more thorough statistical analysis. Nevertheless, this review describes the presence of certain genetic variations, which can be the basis for further development of (targeted) therapies in these gynaecological cancers.

\section{References}

1 Meldrum C, Doyle MA, Tothill RW: Nextgeneration sequencing for cancer diagnostics: a practical perspective. Clin Biochem Rev 2011;32:177-195.

2 De Roock W, Claes B, Bernasconi D, De Schutter J, Biesmans B, Fountzilas G, Kalogeras KT, Kotoula V, Papamichael D, LaurentPuig P, Penault-Llorca F, Rougier P, Vincenzi B, Santini D, Tonini G, Cappuzzo F, Frattini M, Molinari F, Saletti P, De Dosso S, Martini M, Bardelli A, Siena S, Sartore-Bianchi A, Tabernero J, Macarulla T, Di Fiore F, Gangloff AO, Ciardiello F, Pfeiffer P, Qvortrup C, Hansen TP, Van Cutsem E, Piessevaux H, Lambrechts D, Delorenzi M, Tejpar S: Effects of KRAS, BRAF, NRAS, and PIK3CA mutations on the efficacy of cetuximab plus chemotherapy in chemotherapy-refractory metastatic colorectal cancer: a retrospective consortium analysis. Lancet Oncol 2010;11: 753-762.

3 Uji K, Naoi Y, Kagara N, Shimoda M, Shimomura A, Maruyama N, Shimazu K, Kim SJ, Noguchi S: Significance of TP53 mutations determined by next-generation "deep" sequencing in prognosis of estrogen receptorpositive breast cancer. Cancer Lett 2014;342: 19-26.

4 Wheler J, Yelensky R, Falchook G, Kim KB, Hwu P, Tsimberidou AM, Stephens PJ, Hong D, Cronin MT, Kurzrock R: Next generation sequencing of exceptional responders with BRAF-mutant melanoma: implications for sensitivity and resistance. BMC Cancer 2015; 15:61.

5 Torre LA, Bray F, Siegel RL, Ferlay J, LortetTieulent J, Jemal A: Global cancer statistics, 2012. CA Cancer J Clin 2015;65:87-108.

6 Ford CE, Henry C, Llamosas E, Djordjevic A, Hacker N: Wnt signalling in gynaecological cancers: a future target for personalised medicine? Gynecol Oncol 2016;140:345-351.

7 Mukhopadhyay A, Curtin N, Plummer R, Edmondson RJ: PARP inhibitors and epithelial ovarian cancer: an approach to targeted chemotherapy and personalised medicine. BJOG 2011;118:429-432.
8 Hayden JA, Cote P, Bombardier C: Evaluation of the quality of prognosis studies in systematic reviews. Ann Intern Med 2006;144:427437.

9 Hayden JA, van der Windt DA, Cartwright JL, Cote P, Bombardier C: Assessing bias in studies of prognostic factors. Ann Intern Med 2013;158:280-286.

10 Hoogstraat M, Hinrichs JW, Besselink NJ, Radersma-van Loon JH, de Voijs CM, Peeters T, Nijman IJ, de Weger RA, Voest EE, Willems SM, Cuppen E, Koudijs MJ: Simultaneous detection of clinically relevant mutations and amplifications for routine cancer pathology. J Mol Diagn 2015;17:10-18.

11 De Leng WW, Gadellaa-van Hooijdonk CG, Barendregt-Smouter FA, Koudijs MJ, Nijman I, Hinrichs JW, Cuppen E, van Lieshout S, Loberg RD, de Jonge M, Voest EE, de Weger RA, Steeghs N, Langenberg MH, Sleijfer S, Willems SM, Lolkema MP: Targeted next generation sequencing as a reliable diagnostic assay for the detection of somatic mutations in tumours using minimal DNA amounts from formalin fixed paraffin embedded material. PLoS One 2016;11:e0149405.

12 Anglesio MS, Kommoss S, Tolcher MC, Clarke B, Galletta L, Porter H, Damaraju S, Fereday S, Winterhoff BJ, Kalloger SE, Senz J, Yang W, Steed H, Allo G, Ferguson S, Shaw P, Teoman A, Garcia JJ, Schoolmeester JK, Bakkum-Gamez J, Tinker AV, Bowtell DD, Huntsman DG, Gilks CB, McAlpine JN: Molecular characterization of mucinous ovarian tumours supports a stratified treatment approach with HER 2 targeting in 19\% of carcinomas. J Pathol 2013;229:111-120.

13 Bauerschlag DO, Schem C, Weigel MT, Von Kaisenberg C, Strauss A, Bauknecht T, Maass N, Meinhold-Heerlein I: The role of p53 as a surrogate marker for chemotherapeutical responsiveness in ovarian cancer. J Cancer Res Clin Oncol 2010;136:79-88.
14 Despierre E, Yesilyurt BT, Lambrechts S, Johnson N, Verheijen R, van der Burg M, Casado A, Rustin G, Berns E, Leunen K, Amant F, Moerman P, Lambrechts D, Vergote I; EORTC GCG and EORTC GCG Translational Research Group: Epithelial ovarian cancer: rationale for changing the one-fits-all standard treatment regimen to subtype-specific treatment. Int J Gynecol Cancer 2014;24:468-477.

15 Dobrzycka B, Terlikowski SJ, Kinalski M, Kowalczuk O, Niklinska W, Chyczewski L: Circulating free DNA and p53 antibodies in plasma of patients with ovarian epithelial cancers. Ann Oncol 2011;22:1133-1140.

16 Garcia-Dios DA, Lambrechts D, Coenegrachts L, Vandenput I, Capoen A, Webb PM, Ferguson K, Anecs, Akslen LA, Claes B, Vergote I, Moerman P, Van Robays J, Marcickiewicz J, Salvesen HB, Spurdle AB, Amant F: High-throughput interrogation of PIK3CA, PTEN, KRAS, FBXW7 and TP53 mutations in primary endometrial carcinoma. Gynecol Oncol 2013;128:327-334.

17 Gatius S, Velasco A, Azueta A, Santacana M, Pallares J, Valls J, Dolcet X, Prat J, MatiasGuiu X: FGFR2 alterations in endometrial carcinoma. Mod Pathol 2011;24:1500-1510.

18 Jia W, Chang B, Sun L, Zhu H, Pang L, Tao L, Zou H, Du J, Dong Y, Qi Y, Jiang J, Liang W, Li F, Zhao X: REDD1 and p-AKT over-expression may predict poor prognosis in ovarian cancer. Int J Clin Exp Pathol 2014;7:59405949.

19 Wong KK, Tsang YT, Deavers MT, Mok SC, Zu Z, Sun C, Malpica A, Wolf JK, Lu KH, Gershenson DM: BRAF mutation is rare in advanced-stage low-grade ovarian serous carcinomas. Am J Pathol 2010;177:1611-1617.

20 Spaans VM, Trietsch MD, Crobach S, Stelloo E, Kremer D, Osse EM, Haar NT, van Eijk R, Muller S, van Wezel T, Trimbos JB, Bosse T, Smit VT, Fleuren GJ: Designing a highthroughput somatic mutation profiling panel specifically for gynaecological cancers. PLoS One 2014;9:e93451. 
21 Kurman RJ, Shih IM: Pathogenesis of ovarian cancer: lessons from morphology and molecular biology and their clinical implications. Int J Gynecol Pathol 2008;27:151-160.

22 Singer G, Stohr R, Cope L, Dehari R, Hartmann A, Cao DF, Wang TL, Kurman RJ, Shih IM: Patterns of p53 mutations separate ovarian serous borderline tumors and low- and high-grade carcinomas and provide support for a new model of ovarian carcinogenesis: a mutational analysis with immunohistochemical correlation. Am J Surg Pathol 2005;29: 218-224.

23 Cancer Genome Atlas Research Network: Integrated genomic analyses of ovarian carcinoma. Nature 2011;474:609-615.

24 Reles A, Wen WH, Schmider A, Gee C, Runnebaum IB, Kilian U, Jones LA, El-Naggar A, Minguillon C, Schonborn I, Reich O, Kreienberg R, Lichtenegger W, Press MF: Correlation of p53 mutations with resistance to platinum-based chemotherapy and shortened survival in ovarian cancer. Clin Cancer Res 2001;7:2984-2997.

25 Gemignani ML, Schlaerth AC, Bogomolniy F, Barakat RR, Lin O, Soslow R, Venkatraman E, Boyd J: Role of KRAS and BRAF gene mutations in mucinous ovarian carcinoma. Gynecol Oncol 2003;90:378-381.

26 Ratner E, Lu L, Boeke M, Barnett R, Nallur S, Chin LJ, Pelletier C, Blitzblau R, Tassi R, Paranjape T, Hui P, Godwin AK, Yu H, Risch H, Rutherford T, Schwartz P, Santin A, Matloff E, Zelterman D, Slack FJ, Weidhaas JB: A KRAS-variant in ovarian cancer acts as a genetic marker of cancer risk. Cancer Res 2010; 70:6509-6515.

27 Banno K, Yanokura M, Iida M, Masuda K, Aoki D: Carcinogenic mechanisms of endometrial cancer: involvement of genetics and epigenetics. J Obstet Gynaecol Res 2014;40: 1957-1967.

28 Merritt MA, Cramer DW: Molecular pathogenesis of endometrial and ovarian cancer. Cancer Biomark 2010;9:287-305.

29 Mutter GL, Lin MC, Fitzgerald JT, Kum JB, Baak JP, Lees JA, Weng LP, Eng C: Altered PTEN expression as a diagnostic marker for the earliest endometrial precancers. J Natl Cancer Inst 2000;92:924-930.

30 Sun $\mathrm{H}$, Enomoto T, Fujita M, Wada H, Yoshino K, Ozaki K, Nakamura T, Murata Y: Mutational analysis of the PTEN gene in endometrial carcinoma and hyperplasia. Am J Clin Pathol 2001;115:32-38.

31 Salvesen HB, Stefansson I, Kretzschmar EI, Gruber P, MacDonald ND, Ryan A, Jacobs IJ,
Akslen LA, Das S: Significance of PTEN alterations in endometrial carcinoma: a population-based study of mutations, promoter methylation and PTEN protein expression. Int J Oncol 2004;25:1615-1623.

32 Kuhn E, Wu RC, Guan B, Wu G, Zhang J, Wang Y, Song L, Yuan X, Wei L, Roden RB, Kuo KT, Nakayama K, Clarke B, Shaw P, Olvera N, Kurman RJ, Levine DA, Wang TL, Shih IM: Identification of molecular pathway aberrations in uterine serous carcinoma by genome-wide analyses. J Natl Cancer Inst 2012;104:1503-1513.

33 Konopka B, Janiec-Jankowska A, Kwiatkowska E, Najmola U, Bidzinski M, Olszewski W, Goluda C: PIK3CA mutations and amplification in endometrioid endometrial carcinomas: relation to other genetic defects and clinicopathologic status of the tumors. Hum Pathol 2011;42:1710-1719.

34 Rudd ML, Price JC, Fogoros S, Godwin AK, Sgroi DC, Merino MJ, Bell DW: A unique spectrum of somatic PIK3CA (p110alpha) mutations within primary endometrial carcinomas. Clin Cancer Res 2011;17:1331-1340.

35 Fukuchi T, Sakamoto M, Tsuda H, Maruyama K, Nozawa S, Hirohashi S: Beta-catenin mutation in carcinoma of the uterine endometrium. Cancer Res 1998;58:3526-3528.

36 Saegusa M, Hashimura M, Yoshida T, Okayasu I: Beta-catenin mutations and aberrant nuclear expression during endometrial tumorigenesis. Br J Cancer 2001;84:209-217.

37 Bumrungthai S, Munjal K, Nandekar S, Cooper K, Ekalaksananan T, Pientong C, Evans MF: Epidermal growth factor receptor pathway mutation and expression profiles in cervical squamous cell carcinoma: therapeutic implications. J Transl Med 2015;13:244.

38 Iida K, Nakayama K, Rahman MT, Rahman M, Ishikawa M, Katagiri A, Yeasmin S, Otsuki Y, Kobayashi H, Nakayama S, Miyazaki $\mathrm{K}$ : EGFR gene amplification is related to adverse clinical outcomes in cervical squamous cell carcinoma, making the EGFR pathway a novel therapeutic target. Br J Cancer 2011; 105:420-427.

39 Gao J, Aksoy BA, Dogrusoz U, Dresdner G, Gross B, Sumer SO, Sun Y, Jacobsen A, Sinha R, Larsson E, Cerami E, Sander C, Schultz N: Integrative analysis of complex cancer genomics and clinical profiles using the cBioPortal. Sci Signal 2013;6:pl1.

40 Cancer Genome Atlas Research N, Kandoth C, Schultz N, Cherniack AD, Akbani R, Liu Y, Shen H, Robertson AG, Pashtan I, Shen R, Benz CC, Yau C, Laird PW, Ding L, Zhang W, Mills
GB, Kucherlapati R, Mardis ER, Levine DA: Integrated genomic characterization of endometrial carcinoma. Nature 2013;497:67-73.

41 Manzo-Merino J, Contreras-Paredes A, Vazquez-Ulloa E, Rocha-Zavaleta L, FuentesGonzalez AM, Lizano M: The role of signaling pathways in cervical cancer and molecular therapeutic targets. Arch Med Res 2014;45: 525-539.

42 Ledermann JA, Harter P, Gourley C, Friedlander M, Vergote I, Rustin G, Scott C, Meier W, Shapira-Frommer R, Safra T, Matei D, Fielding A, Spencer S, Rowe P, Lowe E, Hodgson D, Sovak MA, Matulonis U: Overall survival in patients with platinum-sensitive recurrent serous ovarian cancer receiving olaparib maintenance monotherapy: an updated analysis from a randomised, placebocontrolled, double-blind, phase 2 trial. Lancet Oncol 2016;17:1579-1589.

43 Grisham RN, Sylvester BE, Won H, McDermott G, DeLair D, Ramirez R, Yao Z, Shen R, Dao F, Bogomolniy F, Makker V, Sala E, Soumerai TE, Hyman DM, Socci ND, Viale A, Gershenson DM, Farley J, Levine DA, Rosen N, Berger MF, Spriggs DR, Aghajanian CA, Solit DB, Iyer G: Extreme outlier analysis identifies occult mitogen-activated protein kinase pathway mutations in patients with low-grade serous ovarian cancer. J Clin Oncol 2015;33:4099-4105.

44 Lheureux S, Oza AM: Endometrial cancertargeted therapies myth or reality? Review of current targeted treatments. Eur J Cancer 2016;59:99-108.

45 Ray-Coquard I, Favier L, Weber B, RoemerBecuwe C, Bougnoux P, Fabbro M, Floquet A, Joly F, Plantade A, Paraiso D, Pujade-Lauraine E: Everolimus as second- or third-line treatment of advanced endometrial cancer: ENDORAD, a phase II trial of GINECO. Br J Cancer 2013;108:1771-1777.

46 Slomovitz BM, Jiang Y, Yates MS, Soliman PT, Johnston T, Nowakowski M, Levenback C, Zhang Q, Ring K, Munsell MF, Gershenson DM, Lu KH, Coleman RL: Phase II study of everolimus and letrozole in patients with recurrent endometrial carcinoma. J Clin Oncol 2015;33:930-936.

47 Stevanovic S, Draper LM, Langhan MM, Campbell TE, Kwong ML, Wunderlich JR, Dudley ME, Yang JC, Sherry RM, Kammula US, Restifo NP, Rosenberg SA, Hinrichs CS Complete regression of metastatic cervical cancer after treatment with human papillomavirus-targeted tumor-infiltrating T cells. J Clin Oncol 2015;33:1543-1550. 